Pacific

Journal of

Mathematics

\title{
BUNDLES WITH SPHERICAL EULER CLASS
}

Luis Guijarro, Thomas Schick, and Gerard Walschap 


\title{
BUNDLES WITH SPHERICAL EULER CLASS
}

\author{
Luis Guijarro, Thomas Schick, and Gerard Walschap
}

In this note, we show that the holonomy group of a Riemannian connection on a $k$-dimensional Euclidean vector bundle is transitive on the unit sphere bundle whenever the Euler class $\chi$ is spherical. We extract several consequences from this, among them that this is always the case as long as $\chi$ does not vanish, and the base of the bundle is simply connected and rationally $(k+1) / 2$-connected.

\section{Introduction and statement of main results.}

Recall that every complete, noncompact Riemannian manifold $M$ with nonnegative sectional curvature contains a compact, totally geodesic (hence also nonnegatively curved) submanifold, called a soul. The soul theorem of Cheeger and Gromoll ([2]) states that $M$ is then diffeomorphic to the normal bundle of the soul in $M$.

When studying the converse, namely the question of whether a vector bundle over a closed nonnegatively curved manifold admits a complete metric which has also nonnegative sectional curvature, there appear to be constraints on the topology of the bundle which imply that any such metric must be rigid. One such is the following:

Definition 1.1. A Euclidean vector bundle with Riemannian connection is said to have transitive holonomy if the holonomy group associated to the connection acts transitively on (any fiber of) the corresponding sphere bundle.

In a previous paper [3], the first and last author showed that if the normal bundle $\nu(S)$ to a soul $S$ of $M$ has transitive holonomy, then:

(1) The exponential map exp $: \nu(S) \rightarrow M$ is a diffeomorphism.

(2) The metric projection $M \rightarrow S$ is smooth.

(3) The ideal boundary $M(\infty)$ of $M$ consists of a single point.

Because of this, the natural goal is to find a topological condition which implies that on a given bundle the holonomy action is always transitive, no matter what the Riemannian metric and Riemannian connection look like. Some results in this direction appear in $[\mathbf{3}]$ and the last section of the paper. What follows is a brief outline of what this article contains; the reader can find the proofs and further discussion in the sections indicated there. All 
vector bundles are Euclidean (i.e., with a metric tensor defined on each fiber), and all connections Riemannian (i.e, compatible with the former).

\section{Spherical Euler class implies transitive holonomy (Section 2).}

Definition 1.2. Let $X$ be a CW-complex and $a \in H^{k}(X, \mathbb{Z})$ a cohomology class. We say $a$ is spherical if there is a map $f: S^{k} \rightarrow X$ such that $f^{*} a \neq$ $0 \in H^{k}\left(S^{k}, \mathbb{Z}\right)$. Otherwise, $a$ is said to be not spherical.

Notice that, in this case, $a$ has infinite order. Moreover, such a cohomology class is never the cup-product of lower dimensional classes. Thus, we get examples of nonvanishing, nonspherical classes, even among differentiable manifolds with positive sectional curvature: They include, for instance, complex or quaternionic projective spaces, since their cohomology rings are polynomial in one generator.

The reason for this definition is given in the following:

Theorem 1.3. Let $E \rightarrow X$ be a $k$-dimensional oriented vector bundle over a $\mathrm{CW}$-complex $X$. If the Euler class $\chi(E)$ is spherical, then the holonomy group of any Riemannian connection on $E$ is transitive.

\section{Conditions ensuring that nonvanishing Euler classes are spherical} (Section 3). In this part, we show that if the base of the bundle is sufficiently connected, then a nonzero Euler class is spherical. More precisely, combining this with the former section, our result says:

Theorem 1.4. Let $X$ be a simply connected finite $\mathrm{CW}$-complex with $H_{j}(X, \mathbb{Q})=0$ for $1 \leq j<n_{1}$, and let $E$ be a $k$-dimensional vector bundle over $X$. Let $n_{2} \geq n_{1}$ be the smallest integer such that $\sum_{j=n_{1}}^{n_{2}} \operatorname{dim} H_{j}(X, \mathbb{Q}) \geq$ 2.

Suppose the rational Euler class of $E$ is nontrivial, i.e.,

$$
0 \neq \chi(E) \in H^{k}(X, \mathbb{Q}),
$$

and assume that either $n_{1}$ is odd and $k \leq n_{1}+n_{2}-1$, or $n_{1}$ is even and $k \leq 2 n_{1}-1$.

Then any Riemannian connection on $E$ has transitive holonomy.

An immediate consequence is contained in the following:

Corollary 1.5. Let $E \rightarrow X$ be a vector bundle satisfying the hypotheses of Theorem 1.4. Then $E$ is irreducible.

Proof. If $E=E_{1} \oplus E_{2}$ is reducible, then a connection on each factor induces a connection on $E$ with non-transitive holonomy.

The upper bound on the rank $k$ of the bundle in Theorem 1.4 is optimal in the sense that the conclusion is no longer true for higher values of $k$. For example, the tangent bundle of the product of 2 -spheres has nonzero 
Euler class, even though the Levi-Civita connection of the standard product metric has non-transitive holonomy. In this particular case, the holonomy action is of course reducible, but one can also find nontransitive irreducible examples by considering the Levi-Civita connection on the tangent bundle of any irreducible, compact, symmetric space of type I of higher rank. These bundles have positive Euler number, and admit complete metrics of nonnegative curvature by an argument similar to that in Example 6.2.

Realization of spherical cohomology classes as Euler classes (Section 4). In this part, we study the question of which cohomology classes can be realized as Euler classes of vector bundles. We are most interested in the existence of spherical Euler classes. The main result is that a finite dimensional CW-complex which has a spherical cohomology class of dimension $k$, also admits a vector bundle of rank $k$ with spherical (in particular nontrivial) Euler class.

This is an immediate corollary of the following theorem, since a spherical cohomology class is never torsion.

Theorem 1.6. Given $k, n \in \mathbb{N}$ with $k$ even, there is an integer $N(k, n)>0$ such that for every $\mathrm{CW}$-complex $X$ of dimension $n$ and every cohomology class $a \in H^{k}(X, \mathbb{Z})$, there is a vector bundle $\xi$ over $X$ with

$$
\chi(\xi)=2 \cdot N(k, n) \cdot a .
$$

One can see that the additional factor is absolutely necessary. In fact, it can be proved that for a sphere $S^{n}$ with $n \neq 2,4$ or 8 , the Euler classes that can appear are even [7].

Remark 1.1. A more general result that includes realization of Pontriagin classes has been obtained by I. Belegradek and V. Kapovitch in [1], Lemma B.1.

Relations between spherical Euler classes and the boundary map of a fibration (Section 5). In [3] it was shown that non-transitive holonomy implies that the boundary maps in the homotopy sequence for the unit sphere bundle vanish. In this paper, we establish that non-transitive holonomy implies that the Euler class is not spherical. In Section 5 of the paper, we show that these two homotopy conditions are actually equivalent.

Theorem 1.7. Let $\xi$ be an oriented rank $k+1$ vector bundle over the base space $B$ with corresponding oriented spherical fibration $E_{1} \rightarrow B$. For a fixed $q \in \mathbb{N}$, if the boundary map $\delta_{q}$ in the long exact homotopy sequence of the fibration $S^{k} \rightarrow E_{1} \rightarrow B$

$$
\pi_{q}\left(E_{1}\right) \rightarrow \pi_{q}(B) \stackrel{\delta_{q}}{\rightarrow} \pi_{q-1}\left(S^{k}\right) \rightarrow \pi_{q-1}\left(E_{1}\right)
$$

is trivial, then for every map $f: S^{q} \rightarrow B$, the pull-back $f^{*}(\chi(\xi))$ of the Euler class of $\xi$ is trivial.

Furthermore, for $q=k+1$ the converse is true. 


\section{Spherical Euler class implies transitive holonomy.}

To prove Theorem 1.3, we begin with a geometric construction that characterizes transitive connections for spheres.

Lemma 2.1. Let $E$ be a Euclidean vector bundle with Riemannian connection over the sphere $S^{k} k \geq 1$, with non-transitive holonomy group. Then $E$ is orientable and admits a global section, i.e., the Euler class $\chi(E)=0$.

Proof. If $E$ is one-dimensional and the Riemannian connection is non-transitive, then the bundle is orientable and flat. An orientable line bundle is trivial, which concludes the proof in this case.

If $\operatorname{dim}(E) \geq 2$, let $p, q$ be two different points on $S^{k}$. Set $U_{p}:=S^{k}-\{q\}$, $U_{q}:=S^{k}-\{p\}$. Consider a partition of unity $\left(\phi_{p}, \phi_{q}\right)$ subordinate to this open cover of $S^{k}$, and choose a unit vector $v \in E_{p}$, the fiber of $E$ over $p$.

Pick a path from $p$ to $q$ and let $v^{\prime}$ be the image of $v$ under parallel transport along this path. Let $H v^{\prime} \subset E_{q}$ be the orbit of $v^{\prime}$ under the holonomy action. By assumption, the holonomy action is not transitive, so this is a proper subset of the unit sphere in $E_{q}$. Choose a unit vector $w \in E_{q}-H v^{\prime}$.

Identify now $\left(U_{p}, p\right)$ and $\left(U_{q}, q\right)$ with the disc $\left(D^{k}, 0\right)$ and define nowhere vanishing vector fields $f_{v}$ on $U_{p}$ and $f_{w}$ on $U_{q}$ by parallel transport of $v$ or $w$, respectively, along radial rays. Set $f:=\phi_{p} f_{v}-\phi_{q} f_{w}$. We claim that this vector field has no zeros, thus proving the lemma because of 9.7 in [5]. Assume the contrary, i.e., that there is a point $x \in S^{k}$ with $\phi_{p}(x) f_{v}(x)=$ $\phi_{q}(x) f_{w}(x)$. Since $f_{v}(x)$ and $f_{w}(x)$ both have length one, and $\phi_{p}(x), \phi_{q}(x) \geq$ 0 , this implies $f_{v}(x)=f_{w}(x)$. But then $w \in H v^{\prime}$, which gives the desired contradiction.

Proof of Theorem 1.3. Fix a Riemannian metric and connection on $E$. Choose $f: S^{k} \rightarrow X$ such that $f^{*}(\chi(E)) \neq 0$. Hence the pull back $f^{*} E$ satisfies $\chi\left(f^{*} E\right)=f^{*}(\chi(E)) \neq 0$. On the other hand, we can pull the metric and connection back to $f^{*} E$, and the corresponding holonomy group is a subgroup of the holonomy group of $E$. If the holonomy of $E$ were nontransitive, the same would then be true for $f^{*} E$. But by Lemma 2.1, this is not possible and the statement follows.

\section{Conditions ensuring that nonvanishing Euler classes are spherical.}

The following condition is useful to check whether a cohomology class is spherical:

Lemma 3.1. A cohomology class $a \in H^{k}(X, \mathbb{Z})$ is spherical if and only if there is a spherical homology class $x \in H_{k}(X, \mathbb{Z})$ such that

$$
\langle a, x\rangle \neq 0 \text {. }
$$


Moreover, this is the case if and only if there is some commutative ring $R$ with unit and $x \in H_{k}(X, R)$ such that $\langle a, x\rangle \neq 0$, where by slight abuse of notation we let a denote the image of $a \in H^{k}(X, \mathbb{Z})$ under the coefficient homomorphism $H^{k}(X, \mathbb{Z}) \rightarrow H^{k}(X, R)$ induced from $\mathbb{Z} \rightarrow R: n \mapsto n \cdot 1$.

Remember that a homology class $x \in H_{k}(X, R)$ is called spherical if it is contained in the image of the Hurewicz homomorphism

$$
\pi_{k}(X) \otimes_{\mathbb{Z}} R \rightarrow H_{k}(X, \mathbb{Z}) \otimes_{\mathbb{Z}} R \rightarrow H_{k}(X, R) ;
$$

in other words, if $x=\sum_{j=1}^{N}\left(f_{j}\right)_{*}\left(\left[S^{k}\right]\right) \cdot r_{j}$ for suitable maps $f_{j}: S^{k} \rightarrow X$ and $r_{j} \in R$.

Proof. Observe that for every $f: S^{k} \rightarrow B$ we have $\left\langle f^{*} a,\left[S^{k}\right]\right\rangle=\left\langle a, f_{*}\left[S^{k}\right]\right\rangle$. Because of the (co)homological structure of $S^{k}$, the left-hand side is zero if and only if $f^{*} a=0$. Together with our definition of a spherical cohomology class, this finishes the proof of the statement for $R=\mathbb{Z}$.

Assume now $\langle a, x\rangle \neq 0$ for some spherical homology class $x \in H_{k}(X, R)$ and some unital ring $R$. Then $x=\sum_{j=1}^{N}\left(f_{j}\right)_{*}\left(\left[S^{k}\right]\right) \cdot r_{j}$ for suitable $f_{j}: S^{k} \rightarrow$ $X$ and $r_{j} \in R$. In other words

$$
0 \neq \sum_{j=1}^{N}\left\langle a,\left(f_{j}\right)_{*}\left[S^{k}\right] \cdot r_{j}\right\rangle=\sum_{j=1}^{N}\left\langle f_{j}^{*} a,\left[S^{k}\right]\right\rangle \cdot r_{j} .
$$

This is only possible if there is some $j \in\{1, \ldots, N\}$ with $\left\langle f_{j}^{*} a,\left[S^{k}\right]\right\rangle \neq 0$; but then $f_{j}^{*} a \neq 0$, i.e., $a$ is spherical.

We now give a general criterion, using Serre theory, which tells us when rational cohomology classes are spherical. This is certainly well-known, but we include a proof for convenience of the reader.

Definition 3.2. Let $\mathcal{C}$ be the class of all finite abelian groups. A homomorphism $u: A \rightarrow B$ is called a $\mathcal{C}$-monomorphism or $\mathcal{C}$-epimorphism if its kernel or cokernel, respectively, belongs to $\mathcal{C}$. It is called a $\mathcal{C}$-isomorphism if it is both a $\mathcal{C}$-monomorphism and a $\mathcal{C}$-epimorphism.

Lemma 3.3. Let $X_{1}, \ldots, X_{N}$ be $N$ simply connected finite $\mathrm{CW}$-complexes such that $\pi_{j}\left(X_{k}\right) \in \mathcal{C}$ for $j<n_{k}, k=1, \ldots, N$, with $n_{1} \leq n_{2} \leq \cdots \leq n_{N}$. Then the inclusion

$$
\alpha: X_{1} \vee \cdots \vee X_{N} \rightarrow X_{1} \times \cdots \times X_{N}
$$

induces $\mathcal{C}$-isomorphisms

$$
\begin{aligned}
& \pi_{j}\left(X_{1} \vee \cdots \vee X_{N}\right) \rightarrow \pi_{j}\left(X_{1} \times \cdots \times X_{N}\right) \\
& \cong \pi_{j}\left(X_{1}\right) \oplus \cdots \oplus \pi_{j}\left(X_{N}\right), \\
& H_{j}\left(X_{1}, \mathbb{Z}\right) \oplus \cdots \oplus H_{j}\left(X_{N}, \mathbb{Z}\right) \\
& \cong H_{j}\left(X_{1} \vee \cdots \vee X_{N}, \mathbb{Z}\right) \rightarrow H_{j}\left(X_{1} \times \cdots \times X_{n}, \mathbb{Z}\right)
\end{aligned}
$$


for $0<j<n_{1}+n_{2}-1$. These maps are $\mathcal{C}$-epimorphisms for $j=n_{1}+n_{2}-1$.

Proof. Assume first that we deal only with two spaces $X$ and $Y$. We know that $H_{j}(X, \mathbb{Z}) \oplus H_{j}(Y, \mathbb{Z}) \cong H_{j}(X \vee Y, \mathbb{Z})$ for all $j \geq 1$, where the isomorphism is induced by the inclusions. Moreover, the Künneth theorem implies $H_{j}(X, \mathbb{Z}) \oplus H_{j}(Y, \mathbb{Z}) \rightarrow H_{j}(X \times Y, \mathbb{Z})$ is a $\mathcal{C}$-isomorphism for $0 \leq j<n_{1}+n_{2}$, where again the map is induced by inclusions. It follows that

$$
H_{j}(\alpha): H_{j}(X \vee Y, \mathbb{Z}) \rightarrow H_{j}(X \times Y, \mathbb{Z})
$$

is a $\mathcal{C}$-isomorphism for $j<n_{1}+n_{2}-1$, and a $\mathcal{C}$-epimorphism for $j=n_{1}+$ $n_{2}-1$. By [6, Theorem 9.6.22] the same can be said of $\pi_{j}(\alpha): \pi_{j}(X \vee Y) \rightarrow$ $\pi_{j}(X \times Y)$, and $\pi_{j}(X \times Y) \cong \pi_{j}(X) \oplus \pi_{j}(Y)$.

The general case follows from this by induction, where we observe that we prove in particular that $\pi_{j}\left(X_{1} \vee \cdots \vee X_{k}\right) \in \mathcal{C}$ for $j<n_{1}$ for all $1 \leq k \leq$ $N$.

We need some knowledge about the homotopy and homology groups of spheres. Therefore recall from [6, Theorems 9.7.7 and 9.7.9]:

Theorem 3.4. The homotopy groups of spheres have the following properties:

- $\pi_{n}\left(S^{n}\right) \cong \mathbb{Z}$.

- $\pi_{j}\left(S^{n}\right)$ if finite if $j \neq n$ and $j \neq 2 n-1$. If $n$ is odd, then $\pi_{2 n-1}\left(S^{n}\right)$ is also finite.

- $H_{n}\left(S^{n}\right) \cong \mathbb{Z}$.

- $H_{j}\left(S^{n}\right)=0$ if $j \neq 0, n$.

Lemma 3.5. Assume $1<n_{1} \leq n_{2} \leq \cdots \leq n_{N}$. Set $n:=2 n_{1}-1$ if $n_{1}$ is even, and $n:=n_{1}+n_{2}-1$ if $n_{1}$ is odd and $N \geq 2$. The Hurewicz homomorphism

$$
\beta_{j}: \pi_{j}\left(S^{n_{1}} \vee \cdots \vee S^{n_{N}}\right) \rightarrow H_{j}\left(S^{n_{1}} \vee \cdots \vee S^{n_{N}}, \mathbb{Z}\right)
$$

is a $\mathcal{C}$-isomorphism for $j<n$ and a $\mathcal{C}$-epimorphism for all $j \geq 1$.

Proof. We have for $j>0$

$$
\begin{aligned}
\pi_{j}\left(S^{n_{1}}\right) \oplus \cdots \oplus \pi_{j}\left(S^{n_{N}}\right) \stackrel{\alpha_{j}}{\longrightarrow} \pi_{j}\left(S^{n_{1}} \vee \cdots \vee S^{n_{N}}\right) \\
\quad \stackrel{\beta_{j}}{\longrightarrow} H_{j}\left(S^{n_{1}} \vee \cdots \vee S^{n_{N}}\right) \cong H_{j}\left(S^{n_{1}}\right) \oplus \cdots \oplus H_{j}\left(S^{n_{N}}\right) .
\end{aligned}
$$

The composition is just the direct sum of the Hurewicz homomorphisms for the different spheres. By Lemma $3.3, \alpha_{j}$ is a $\mathcal{C}$-isomorphism for $j<$ $n_{1}+n_{2}-1$. The explicit computation of the rational homotopy groups (and the homology) of spheres as described in Theorem 3.4 (compare [6, Theorems 9.7.7 and 9.7.9]) implies that the composition is a $\mathcal{C}$-isomorphism for $j<n$. Since $n \leq n_{1}+n_{2}-1, \beta_{j}$ is a $\mathcal{C}$-isomorphism for $j<n$. Obviously, 
the composition is a $\mathcal{C}$-epimorphism for every $j>0$, therefore the same is true for $\beta_{j}$.

Proposition 3.6. Let $X$ be a simply connected finite $\mathrm{CW}$-complex. Assume $H_{j}(X, \mathbb{Z}) \in \mathcal{C}$ for $1 \leq j<n_{1}$. Let $n_{2}$ be the smallest positive integer such that $\sum_{j=n_{1}}^{n_{2}} \operatorname{rank} H_{j}(X, \mathbb{Z}) \geq 2$. Set $n:=2 n_{1}-1$ if $n_{1}$ is even, and $n:=n_{1}+n_{2}-1$ if $n_{1}$ is odd.

Then the Hurewicz homomorphism

$$
\beta_{k}(X): \pi_{k}(X) \rightarrow H_{k}(X, \mathbb{Z})
$$

is a $\mathcal{C}$-isomorphism for $k<n$, and a $\mathcal{C}$-epimorphism for $k=n$.

Proof. By [6, Corollary 9.6.16], $\pi_{k}(X)$ is a finitely generated abelian group for every $k \in \mathbb{N}$, since the same clearly is true for $H_{k}(X, \mathbb{Z})$. Set $b_{k}:=$ $\operatorname{rank}\left(\pi_{k}(X)\right)$, and let $Y_{k}:=S^{k} \vee \cdots \vee S^{k}$ be the wedge of $b_{k}$ copies of $S^{k}$, and $Y:=Y_{n_{1}} \vee \cdots \vee Y_{n}$. For each $n_{1} \leq k \leq n$, pick representatives $f_{k, j}: S^{k} \rightarrow X$ $\left(j=1, \ldots, b_{k}\right)$ of elements of $\pi_{k}(X)$ which generate a subgroup of finite index in $\pi_{k}(X)$ and represent them by maps $S^{k} \rightarrow X$. They fit together to define maps $f_{k}: Y_{k} \rightarrow X$ and $f: Y \rightarrow X$ such that $\pi_{k}\left(f_{k}\right)$ is a $\mathcal{C}$-isomorphism. We get the commutative square

$$
\begin{array}{cr}
\pi_{k}(Y) \stackrel{\pi_{k}(f)}{\longrightarrow} & \pi_{k}(X) \\
\downarrow \beta_{k}(Y) & \\
H_{k}(Y, \mathbb{Z}) \stackrel{H_{k}(X)}{\stackrel{H_{k}(f)}{\longrightarrow}} H_{k}(X, \mathbb{Z}) .
\end{array}
$$

We claim that $\pi_{k}(f), \beta_{k}(Y)$ and $H_{k}(f)$ all are $\mathcal{C}$-isomorphisms for $k<n$ and $\mathcal{C}$-epimorphisms for $k=n$. By a (very easy) diagram chase the same is then true for $\beta_{k}(X)$ and the assertion is proved.

The assertion is true for $\beta_{k}(Y)$ because of Lemma 3.5. Since $Y$ and $X$ both are simply connected, the generalized Hurewicz theorem [6, Theorem 9.6.22] implies that if $\pi_{k}(f)$ is a $\mathcal{C}$-isomorphism for $k<n$ and a $\mathcal{C}$-epimorphism for $k=n$, then the same is true for $H_{k}(f)$.

It remains to prove the statement for $\pi_{k}(f)$.

By construction, for $n_{1} \leq k \leq n$,

$$
\bigoplus_{j=1}^{b_{k}} \pi_{k}\left(S^{k}\right) \stackrel{\oplus \pi_{k}\left(f_{k, j}\right)}{\longrightarrow} \pi_{k}(X)
$$

is a $\mathcal{C}$-isomorphism. By Lemma 3.3, and since most homotopy groups of spheres are finite by Theorem 3.4,

$$
\pi_{k}(Y) \rightarrow \bigoplus_{j=n_{1}}^{n} \bigoplus_{l=1}^{b_{j}} \pi_{k}\left(S^{j}\right) \rightarrow \bigoplus_{l=1}^{b_{k}} \pi_{k}\left(S^{k}\right)
$$


is a $\mathcal{C}$-isomorphism for $k<n$ and a $\mathcal{C}$-epimorphism for $k=n$, where the first map is the direct sum of all maps induced from the collapse to the different factors, and the second one is the projection onto the corresponding factors in the direct sum. Consequently, the composition

$$
\pi_{k}(f): \pi_{k}(Y) \rightarrow \bigoplus_{j=1}^{b_{k}} \pi_{k}\left(S^{k}\right) \stackrel{\oplus \pi_{k}\left(f_{k, j}\right)}{\longrightarrow} \pi_{k}(X)
$$

is a $\mathcal{C}$-isomorphism for $j<n$ and a $\mathcal{C}$-epimorphism for $j=n$.

This concludes the proof.

Proposition 3.7. Assume $X$ is a simply connected finite $\mathrm{CW}$-complex such that $H_{j}(X, \mathbb{Q})=0$ for $1 \leq j \leq n_{1}$. Let $n_{2}$ be the smallest integer such that $\sum_{j=n_{1}}^{n_{2}} \operatorname{dim} H_{j}(X, \mathbb{Q}) \geq 2$.

Let $n:=2 n_{1}-1$ if $n_{1}$ is even, and $n:=n_{1}+n_{2}-1$ if $n_{1}$ is odd.

If $k \leq n$ and $x \in H_{k}(X, \mathbb{Q})$ or $a \in H^{k}(X, \mathbb{Q})$ then $x$ or $a$, respectively, is spherical.

Proof. Since $\mathbb{Q}$ is a field, the statement about cohomology classes follows immediately from the statement about homology classes. For the latter, we claim that under the given conditions the Hurewicz homomorphism

$$
\pi_{k}(X) \otimes_{\mathbb{Z}} \mathbb{Q} \rightarrow H_{k}(X, \mathbb{Z}) \otimes_{\mathbb{Z}} \mathbb{Q} \cong H_{k}(X, \mathbb{Q})
$$

is an isomorphism for $k<n$ and an epimorphism for $k=n$, which implies the claim.

But this statement is just a reformulation of Proposition 3.6, since all the groups in question are finitely generated.

Theorem 1.4 is an immediate consequence of Proposition 3.7 and Theorem 1.3.

Example 3.8. Let $V_{2 k, k}$ denote the Stiefel manifold of orthonormal $k$-frames in $\mathbb{R}^{2 k}$, where $k$ is any even integer. $V_{2 k, k}$ is the principal $S O(k)$ bundle of the canonical rank $k$ bundle $\gamma^{k}$ over the Grassmannian $G_{2 k, k}$. Consider the orthogonal complement $\gamma^{k \perp}$ defined by the sequence

$$
0 \rightarrow \gamma^{k} \rightarrow G_{2 k, k} \times \mathbb{R}^{2 k} \rightarrow \gamma^{k \perp} \rightarrow 0,
$$

and let $\xi$ denote the pullback of $\gamma^{k \perp}$ via the bundle projection $\pi: V_{2 k, k} \rightarrow$ $G_{2 k, k}$.

Observe that $\xi$ is stably trivial: Indeed, $\xi \oplus \pi^{*} \gamma^{k}=V_{2 k, k} \times \mathbb{R}^{2 k}$, and $\pi^{*} \gamma^{k}$ is a trivial bundle, since its total space may be identified with $V_{2 k, k} \times \mathbb{R}^{k}$ by mapping $(b, v) \in V_{2 k, k} \times \gamma^{k}$ to $\left(b,[v]_{b}\right) \in V_{2 k, k} \times \mathbb{R}^{k}$, where $[v]_{b}$ denotes the coordinate vector of $v$ in the basis $b$.

Finally, we claim that $\xi$ is irreducible, and in fact, that any connection on $\xi$ is transitive. By Theorem 1.3, it suffices to show that the Euler class of $\xi$ is spherical. For this, fix an orthonormal $(k-1)$-frame $\left(v_{1}, \ldots, v_{k-1}\right)$ of 
$\mathbb{R}^{2 k}$, and consider the $k$-dimensional sphere $S^{k}$ consisting of all unit vectors $v$ in $\mathbb{R}^{2 k}$ that are orthogonal to the $v_{i}$. $S^{k}$ embeds naturally into $V_{2 k, k}$ by mapping $v \in S^{k}$ to $\imath(v):=\left(v_{1}, \ldots, v_{k-1}, v\right)$.

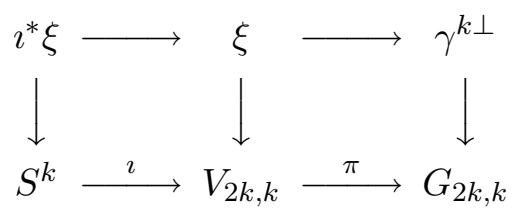

Now $\imath^{*} \xi$ has nonzero Euler characteristic since it is the tangent bundle of the even-dimensional sphere $S^{k}$ : If $P$ denotes the span of the $v_{i}$ 's, then $\imath\left(S^{k}\right)$ is just the unit sphere in $P^{\perp}$, and the fiber over $v \in S^{k}$ consists of all vectors in $P^{\perp}$ that are orthogonal to $v$, i.e., the tangent space of $S^{k}$ at $v$.

\section{Realization of Euler classes.}

Recall that in this section, our objective is to show that a finite dimensional CW-complex which has a spherical cohomology class of dimension $k$ admits a vector bundle of rank $k$ with spherical (in particular nontrivial) Euler class, and hence always having transitive holonomy. Recall also that because a spherical cohomology class has no torsion, it is enough to show that there is a vector bundle whose Euler class is a multiple of the originally given class (this actually constitutes the formulation of Theorem 1.6).

For a CW-complex $X, H^{k}(X, \mathbb{Z})$ corresponds to homotopy classes of maps from $X$ to the Eilenberg-McLane space $K(\mathbb{Z}, k)$. Remember that $K(\mathbb{Z}, k)$ is defined by the property that $\pi_{j}(K(\mathbb{Z}, k))=0$ if $j \neq k$, and $\pi_{k}(K(\mathbb{Z}, k)) \cong \mathbb{Z}$. This defines $K(\mathbb{Z}, k)$ uniquely up to weak homotopy equivalence. Observe (using the Hurewicz homomorphism) that $H^{k}(K(\mathbb{Z}, k), \mathbb{Z}) \cong \mathbb{Z}$ with generator $u$. The correspondence mentioned above is defined as follows: To $f: X \rightarrow K(\mathbb{Z}, k)$ we associate $f^{*} u$.

Because of these observations, we first construct vector bundles over $K(\mathbb{Z}, k)$ :

Theorem 4.1. Given $k, m \in \mathbb{N}$, let $K(\mathbb{Z}, k)^{m}$ be the $m$-skeleton of $K(\mathbb{Z}, k)$, with inclusion map $i: K(\mathbb{Z}, k)^{m} \hookrightarrow K(\mathbb{Z}, k)$. If $k$ is even then there is $N(k, m)>0$ and a vector bundle $\xi_{k, m}$ over $K(\mathbb{Z}, k)^{m}$ with $\chi\left(\xi_{k, m}\right)=2$. $N(k, m) \cdot i^{*} u$.

Theorem 1.6 follows immediately from Theorem 4.1. Assume $a \in H^{k}(X, \mathbb{Z})$ and $a=f^{*} u$ for a suitable $f: X \rightarrow K(\mathbb{Z}, k)$. Since $X$ is $m$-dimensional, we may assume that the image of $f$ is contained in $K(\mathbb{Z}, k)^{m}$, i.e., we have a factorization $f=i \circ f^{\prime}$ with $f^{\prime}: X \rightarrow K(\mathbb{Z}, k)^{m}$. Then

$$
\chi\left(\left(f^{\prime}\right)^{*}\left(\xi_{k, m}\right)\right)=\left(f^{\prime}\right)^{*}\left(N(k, m) i^{*} u\right)=N(k, m) f^{*} u=N(k, m) \cdot a
$$

as we had to prove. 
We are going to obtain the bundles over $K(\mathbb{Z}, k)^{m}$ by induction on $m$. This will be done in two steps: We first show that we can pull back the tangent bundle from $S^{k}$ with a suitable map $K(\mathbb{Z}, k)^{m} \rightarrow S^{k}$, as long as $m \leq 2 k-1$. Starting with the map $K(\mathbb{Z}, k)^{2 k-1} \rightarrow S^{k}$ we will then construct suitable maps to $B S O(k)$, the classifying space for oriented vector bundles of rank $k$.

Obviously, this involves classical obstruction theory. To make these obstructions vanish, we can use composition with the map $\mu_{N}: K(\mathbb{Z}, k) \rightarrow$ $K(\mathbb{Z}, k)$ which induces multiplication by $N$ on $H^{k}(K(\mathbb{Z}, k), \mathbb{Z})$ (because of the classifying property, there is a map $\mu_{N}$, unique up to homotopy, which has this property).

Proposition 4.2. Let $A$ be a finite abelian group of order $N$. Then the map $\mu_{N}: K(\mathbb{Z}, k) \rightarrow K(\mathbb{Z}, k)$ induces the zero map on $H^{i}(K(\mathbb{Z}, k), A)$ for all $i>0$.

Proof. This can be deduced from explicit computations of the cohomology of $K(\mathbb{Z}, k)$ (compare e.g., [4, Theorem 6.12]) and by considering the natural long exact cohomology sequence of a short exact sequence of coefficients.

We prefer to give an independent proof by induction on $k$. Observe that $K(\mathbb{Z}, 1)=S^{1}$, so the statement is fulfilled for $S^{1}$ : Indeed, all higher cohomology groups vanish, and by the universal coefficient theorem it is exactly the defining property of $\mu_{N}$ to induce multiplication by $N$ on $H^{1}(K(\mathbb{Z}, 1), A)$. Since the order of $A$ is $N$, this is the zero map.

We also know that $K(\mathbb{Z}, 2)=\mathbb{C P}^{\infty}$, and the ring $H^{*}\left(\mathbb{C P}^{\infty}, \mathbb{Z}\right)$ is a polynomial ring generated by $u \in H^{2}\left(\mathbb{C P}^{\infty}, \mathbb{Z}\right)$. Consequently $H^{2 i}\left(\mu_{N}\right)$ is multiplication by $N^{i}$. Again, the universal coefficient theorem and the fact that $|A|=N$ implies that $\mu_{N}$ induces the zero map on $H^{j}\left(\mathbb{C P}^{\infty}, A\right)$ for $j>0$.

If $k \geq 2$ we have the path fibration $\Omega K(\mathbb{Z}, k) \rightarrow P K(\mathbb{Z}, k) \rightarrow K(\mathbb{Z}, k)$. Here, for a fixed basepoint $* \in K(\mathbb{Z}, k), P K(\mathbb{Z}, k):=\{f:[0,1] \rightarrow K(\mathbb{Z}, k) \mid$ $f(0)=*\}$, and $\Omega K(\mathbb{Z}, k):=\{f:[0,1] \rightarrow K(\mathbb{Z}, k) \mid f(0)=f(1)=*\}$ with the compact-open topology. The map $\mu_{N}$ evidently induces a self map $U_{n}$ of this fibration. Observe that $P K(\mathbb{Z}, k)$ is contractible. The long exact homotopy sequence of this fibration shows that the fiber $\Omega K(\mathbb{Z}, k)$ is homotopy equivalent to $K(\mathbb{Z}, k-1)$. Using the Hurewicz homomorphism and naturality of the long exact homotopy sequence we see that the restriction of $U_{N}$ to the fiber $K(\mathbb{Z}, k-1)$ induces multiplication by $N$ on $\pi_{k-1}(K(\mathbb{Z}, k-$ $1)$ ) and by the universal coefficient theorem also on $H^{k-1}(K(\mathbb{Z}, k-1), \mathbb{Z})$. Therefore, it coincides (up to homotopy) with the map $\mu_{N}$ of $K(\mathbb{Z}, k-1$ ) and we can apply the induction hypothesis.

Consider now the Leray-Serre spectral sequence computing cohomology with coefficients in $A$ of the path fibration $P K(\mathbb{Z}, k) \rightarrow K(\mathbb{Z}, k)$. Since $k \geq 2$, the base space is simply connected, and therefore the $E_{2}$-term of this spectral sequence is $E_{2}^{p, q}=H^{p}\left(K(\mathbb{Z}, k) ; H^{q}(K(\mathbb{Z}, k-1), A)\right)$, with untwisted 
coefficients. The Leray-Serre spectral sequence is natural with respect to maps of fibrations, and the induced map on the $E_{2}$-term is given by the induced maps on the cohomology of the fiber and the base. Accordingly, by the induction hypothesis, $U_{N}$ induces the zero map on $E_{2}^{p, q}$ as long as $q>0$, and therefore also on the succesive subquotients $E_{r}^{p, q}$ for $q>0$ and $r \geq 2$. Since the total space $P K(\mathbb{Z}, k)$ is contractible, $H^{p}(P K(\mathbb{Z}, k) ; A)=0$ for $p>0$. It follows that for $p>0$ each element of $E_{2}^{p, 0}=H^{p}(K(\mathbb{Z}, k) ; A)$ eventually is in the image of a differential in the spectral sequence, and therefore $U^{*} H^{p}(K(\mathbb{Z}, k) ; A)$ is in the image of $U^{*} E_{r}^{p-r, q+r-1}=0$ for some $r \geq 2$, in other words $U^{*} H^{p}(K(\mathbb{Z}, k) ; A)=0$ for $p>0$. This implies that all classes in the image $\left(U_{N}\right)^{*} H^{p}(K(\mathbb{Z}, k), A)$ are permanent cocycles, and therefore give certain contributions to $H^{p}(P K(\mathbb{Z}, k), A)$. Since the total space is contractible, the latter is zero if $p>0$, and it follows that $\left(U_{N}\right)^{*} H^{p}(K(\mathbb{Z}, k), A)=0$ for $p>0$.

The proposition follows by induction.

Before continuing, we provide some background on the kind of obstruction theory that will be used. The relevant material can be found in $[\mathbf{8}$, Chaper V.5]. Let $X$ be a CW-complex with $k$-skeleton $X^{k}$. Assume $Y$ is another space and we have a map $f: X^{k} \rightarrow Y$. Our goal is to extend $f$ to the $(k+1)$-skeleton $X^{k+1}$. Each $(k+1)$-cell of $X$ is attached to $X^{k}$ via a map of the boundary $S^{k}$ of the cell to $X^{k}$, representing an element $\alpha \in \pi_{k}\left(X^{k}\right)$. The map $f$ is prescribed on the boundary, and can be extended over the $(k+1)$-cell if and only if $f_{*} \alpha=0 \in \pi_{k}(Y)$.

Assigning to each $(k+1)$-cell the corresponding element $f_{*} \alpha$ produces a $(k+1)$-cocycle $o(f)$ on $X$ with values in $\pi_{k}(Y)$. If $X$ is simply connected, this system of coefficients is untwisted and we get an obstruction $[o(f)] \in$ $H^{k+1}\left(X, \pi_{k}(Y)\right)$. As we have seen, the map $f$ can be extended to $X^{k+1}$ if and only if the corresponding cocycle $o(f)$ is identically equal to zero. If (and only if) the cohomology class $[o(f)] \in H^{k+1}\left(X, \pi_{k}(Y)\right)$ vanishes, then we can change $f$ on the $k$-cells (without changing it on $X^{k-1}$ ) in such a way that the new map $f^{\prime}$ has an extension to $X^{k+1}$.

Let $g: X \rightarrow X$ be a self map. Then $o(f g)=g^{*} o(f) \in H^{k+1}\left(X, \pi_{k}(Y)\right)$.

We can use this to produce our maps from $K(\mathbb{Z}, k)^{n}$ to $S^{k}$ or to $B S O(k)$, respectively. We start with a lemma:

Lemma 4.3. If $n<2 k$ then there is an integer $N(k, n)>0$ and a map

$$
f_{n}: K(\mathbb{Z}, k)^{n} \rightarrow S^{k}
$$

such that

$$
f^{*}\left[S^{k}\right]=N(k, n) \cdot i^{*} u,
$$

where $\left[S^{k}\right]$ is a generator of $H^{k}\left(S^{k}, \mathbb{Z}\right) \cong \mathbb{Z}$. 
Proof. The statement is trivial for $k=1$, since $K(\mathbb{Z}, 1)=S^{1}$. Assume therefore that $k>1$. We can choose a model of $K(\mathbb{Z}, k)$ such that

$$
K(\mathbb{Z}, k)^{k}=K(\mathbb{Z}, k)^{k+1}=S^{k} .
$$

Consequently, the identity map gives a map

$$
f_{k+1}: K(\mathbb{Z}, k)^{k+1} \rightarrow S^{k}
$$

which pulls the generator of $H^{k}\left(S^{k}\right)$ back to $i^{*} u$. Now observe that for $k<j<2 k-1$ the homotopy groups $\pi_{j}\left(S^{k}\right)$ are finite (Theorem 3.4, compare [6, Theorems 9.7.7 and 9.7.9]). Assume by induction that for $k<n<2 k-1$ we have $f_{n}: K(\mathbb{Z}, k)^{n} \rightarrow S^{n}$ such that $f_{n}^{*}\left[S^{k}\right]=N(k, n)$. $i^{*} u$. We would like to extend this map to $K(\mathbb{Z}, k)^{n+1}$. This gives an obstruction $\left[o\left(f_{n}\right)\right] \in H^{n+1}\left(K(\mathbb{Z}, k), \pi_{n}\left(S^{k}\right)\right)$ (with untwisted coefficients, since $\pi_{1}(K(\mathbb{Z}, k))=\{1\}$ for $\left.k>1\right)$. By Proposition $4.2 \mu_{\left|\pi_{n}\left(S^{k}\right)\right|}$ induces the zero map on $H^{n+1}\left(K(\mathbb{Z}, k), \pi_{n}\left(S^{k}\right)\right)$. Consequently, the obstruction $\left[o\left(f_{n} \circ \mu_{\left|\pi_{n}\left(S^{k}\right)\right|}\right)\right]=\mu_{\left|\pi_{n}\left(S^{k}\right)\right|}^{*}\left[o\left(f_{n}\right)\right]=0$. This means that we find a map $f^{\prime}$ which coincides with $f_{n} \circ \mu_{\left|\pi_{n}\left(S^{k}\right)\right|}$ on $K(\mathbb{Z}, k)^{n-1}$ and extends to $f_{n+1}: K(\mathbb{Z}, k)^{n+1} \rightarrow S^{k}$. It only remains to observe that since $n>k, f^{\prime}$ coincides with $f_{k} \circ \mu_{\left|\pi_{n}\left(S^{k}\right)\right|}$ on $K(\mathbb{Z}, k)^{k}=K(\mathbb{Z}, k)^{k+1}$. This implies that

$$
f_{n+1}^{*}\left[S^{k}\right]=\left(f^{\prime}\right)^{*}\left[S^{k}\right]=\left(f_{n} \circ \mu_{\left|\pi_{n}\left(S^{k}\right)\right|}\right)^{*}\left[S^{k}\right]=N(k, n) \cdot\left|\pi_{n}\left(S^{k}\right)\right| \cdot i^{*} u .
$$

Remark 4.1. The proof of Lemma 4.3 shows that we can choose $N(k, n)=$ $\prod_{j=k+1}^{n-1}\left|\pi_{j}\left(S^{k}\right)\right|$ for $k \leq n<2 k$.

Lemma 4.4. If $j \geq 2 k-1$, then $\pi_{j}(B S O(k))$ is finite.

Proof. Since $B S O(1)$ is contractible, the statement follows trivially for $k=$ 1.

Assume therefore that $k>1$. We have the principal fibration

$$
S O(k) \rightarrow E S O(k) \rightarrow B S O(k)
$$

with $\operatorname{ESO}(k)$ contractible. It follows from the long exact homotopy sequence of this fibration that $\pi_{j}(B S O(k)) \cong \pi_{j-1}(S O(k))$. We want to prove by induction that $\pi_{j}(S O(k))$ is finite for $j \geq 2 k-2$. We know that $S O(2)=S^{1}$. Consequently, using Theorem $3.4, \pi_{j}(S O(2))=\{0\}$ for $j \geq 2=2 * 2-2$, and the statement follows for $k=2$. Consider now the principal fibration

$$
S O(k-1) \rightarrow S O(k) \rightarrow S^{k-1} .
$$

We obtain exact sequences

$$
\pi_{j}(S O(k-1)) \rightarrow \pi_{j}(S O(k)) \rightarrow \pi_{j}\left(S^{k-1}\right) .
$$


If $k>3$ then by induction, $\pi_{j}(S O(k-1))$ is finite for $j \geq 2 k-2>2(k-1)-2$. Moreover, by Theorem 3.4, $\pi_{j}\left(S^{k-1}\right)$ is finite for $j \geq 2 k-2$. The exact sequence implies that $\left|\pi_{j}(S O(k))\right|<\infty$ for $j \geq 2 k-2$. This concludes the proof.

Remark 4.2. The proof of Lemma 4.4 shows that the orders of the homotopy groups of spheres give an upper bound for $\left|\pi_{n}(S O(k))\right|$. For $n \geq 2 k-1$,

$$
\left|\pi_{n}(B S O(k))\right| \leq \prod_{j=2}^{k-1}\left|\pi_{n-1}\left(S^{j}\right)\right| .
$$

Proposition 4.5. Assume that $k \in \mathbb{N}$ is even. Let $e \in H^{k}(B S O(k), \mathbb{Z})$ be the Euler class of the universal bundle. For every $n \geq k$, there is an integer $N(k, n)>0$ and a map

$$
g_{n}: K(\mathbb{Z}, k)^{n} \rightarrow B S O(k)
$$

such that

$$
g_{n}^{*} e=2 \cdot N(k, n) \cdot i^{*} u .
$$

Proof. Let $t: S^{k} \rightarrow B S O(k)$ be the classifying map of the tangent bundle of $S^{k}$. If $n<2 k$, we constructed $f_{n}: K(\mathbb{Z}, k)^{n} \rightarrow S^{k}$ in Lemma 4.3. In this case, set $g_{n}:=t \circ f_{n}$. Since $\chi\left(S^{k}\right)=2, g_{n}^{*} e=2 \cdot f_{n}^{*}\left[S^{k}\right]=2 N(k, n) i^{*} u$, as required, with $N(k, n)>0$ as in Lemma 4.3.

By induction, we now want to construct $g_{n}: K(\mathbb{Z}, k)^{n} \rightarrow B S O(k)$ for $n \geq 2 k$ with the required properties, using the map $g_{2 k-1}$ just obtained. This is done exactly as in the proof of Lemma 4.3, using the fact that $\pi_{n}(B S O(k))$ is finite for $n \geq 2 k-1$ by Lemma 4.4 .

Remark 4.3. As above, we can give an estimate on $N(k, n)$ in Proposition 4.5. We had $N(k, n)=\prod_{j=k+1}^{n-1}\left|\pi_{j}\left(S^{k}\right)\right|$ if $k \leq n \leq 2 k-1$. For $n \geq 2 k$ we get

$$
N(k, n) \leq \prod_{j=k+1}^{2 k-2}\left|\pi_{j}\left(S^{k}\right)\right| \cdot \prod_{j=2 k-2}^{n-2} \prod_{i=2}^{k-1}\left|\pi_{j}\left(S^{i}\right)\right| .
$$

Of course, since we can pull back the universal bundle from $B S O(k)$ via $g_{k}$, Proposition 4.5 is equivalent to Theorem 4.1. This concludes the proof of all the statements in this section, as well as of Theorem 1.6.

\section{Spherical Euler class and the boundary map of a fibration.}

To prove Theorem 1.7, assume we have an element of $\pi_{q}(B)$ represented by $f: S^{q} \rightarrow B$. Pulling back the fibration via $f$, we obtain a commutative 
diagram of long exact homotopy sequences

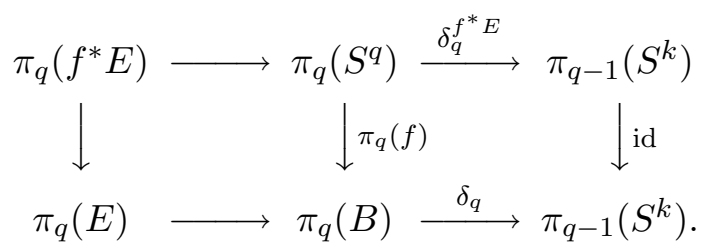

We may assume that $q \geq 1$. Because of commutativity, $\delta_{q}\left(\left[f: S^{q} \rightarrow B\right]\right)$ is trivial if and only if $\delta_{q}^{f^{*} E}\left(\left[\mathrm{id}: S^{q} \rightarrow S^{q}\right]\right)$ is trivial. Since $\pi_{q}\left(S^{q}\right)$ is cyclic and generated by $\left[\mathrm{id}_{S^{q}}\right]$, this is true if and only if $\delta_{q}^{f^{*} E}$ is the zero map, i.e., $\pi_{q}\left(f^{*} E\right) \rightarrow \pi_{q}\left(S^{q}\right)$ is surjective. In other words, $\delta_{q}\left(f: S^{q} \rightarrow B\right)$ is trivial if and only if we find a map $g: S^{q} \rightarrow f^{*} E$ such that $p \circ g$ is homotopic to $\operatorname{id}_{S^{q}}$, where $p: f^{*} E \rightarrow S^{q}$ is the bundle projection. Because of the homotopy lifting property of the fibration $p: f^{*} E \rightarrow S^{q}$, we can replace each such $g$ by a homotopic map $g^{\prime}$ so that $p \circ g^{\prime}=\mathrm{id}$. The existence of such a $g^{\prime}$ means that the fibration has a section, and thus the vanishing of the Euler class $\chi\left(f^{*} \xi\right)=f^{*} \chi(\xi)$. Finally, the last sentence is an if and only if when $q=k+1$.

Corollary 5.1. For an oriented Riemannian vector bundle $\xi$ of rank $k+1$ over a CW-complex $X$, if the long exact homotopy sequence of the unit sphere bundle $E_{1}$ of $\xi$ splits into short exact sequences

$$
0 \rightarrow \pi_{q}\left(S^{k-1}\right) \rightarrow \pi_{q}\left(E_{1}\right) \rightarrow \pi_{q}(X) \rightarrow 0, \quad q \in \mathbb{N}
$$

then the Euler class $\chi(\xi)$ is not spherical.

\section{Spherical Euler class and metrics of nonnegative curvature.}

As pointed out in the introduction, the above results imply that if $M$ is an open manifold with nonnegative sectional curvature, and the normal bundle of a soul in $M$ has spherical Euler class, then the metric on $M$ is quite rigid. This follows from the following theorem proved in [3]:

Theorem 6.1. Let $M$ denote a complete noncompact manifold of nonnegative sectional curvature with soul $S$. If the normal bundle $\nu(S)$ of $S$ in $M$ has transitive holonomy group, then:

(1) The exponential map $\exp : \nu(S) \rightarrow M$ is a diffeomorphism.

(2) The metric projection $M \rightarrow S$ is a $\mathcal{C}^{\infty}$ Riemannian submersion.

(3) The ideal boundary $M(\infty)$ consists of a single point.

For purposes of illustration, we discuss a typical example, cf. also [3]:

Example 6.2. Let $E$ denote the total space of the canonical quaternion line bundle over $\mathbb{H} \mathbb{P}^{n}$, whose fiber over $l \in \mathbb{H} \mathbb{P}^{n}$ consists of $l$ itself. $E$ admits a complete metric of nonnegative sectional curvature because it can be realized 
as a quotient of the nonnegatively curved manifold $S^{4 n+3} \times \mathbb{R}^{4}$ (with the standard product metric) by the group $S^{3}$ of isometries acting diagonally, via the Hopf fibration on the left factor, and by quaternion multiplication on the right.

We claim that any metric of nonnegative curvature on $E$ for which the zero-section is a soul satisfies the conclusions of Theorem 6.1: Indeed, the normal bundle of the soul is then equivalent to the canonical bundle, and its Euler class is nonzero (it is in fact a generator of $H^{4}\left(\mathbb{H} \mathbb{P}^{n}\right)$, see [5]). For $n=1$, the claim now follows from Lemma 2.1, and for $n>1$ from Theorem 1.4.

\section{References}

[1] I. Belegradek and V. Kapovitch, Obstructions to nonnegative curvature and rational homotopy theory. Preprint, 2001.

[2] J. Cheeger and D. Gromoll, On the structure of complete manifolds of nonnegative curvature, Ann. of Math., 96 (1972), 413-443, MR 46 \#8121, Zbl 0246.53049.

[3] L. Guijarro and G. Walschap, Transitive holonomy group and rigidity in nonnegative curvature, Math. Z., 237 (2001), 251-257, CMP 1838310.

[4] J. McCleary, User's Guide to Spectral Sequences, Publish or Perish Inc., Wilmington, Del., 1985, MR 87f:55014, Zbl 0577.55001.

[5] J.W. Milnor and J.D. Stasheff, Characteristic Classes, Annals of Mathematics Studies, 76, Princeton University Press, Princeton, NJ, 1974, MR 55 \#13428, Zbl 0298.57008.

[6] E.H. Spanier, Algebraic Topology, Springer-Verlag, New York, 1981, corrected reprint, MR 83i:55001, Zbl 0477.55001.

[7] G. Walschap, The Euler class as a cohomology generator, to appear in Illinois Mathematical Journal.

[8] G.W. Whitehead, Elements of Homotopy Theory, Springer-Verlag, New York, 1978, MR 80b:55001, Zbl 0406.55001.

Received March 7, 2001 and revised September 17, 2001.

Departamento de Geometría y Topología

Universidad Complutense DE MadRid

CiUdad Universitaria S/N

28040 MADRID, SPAIN

E-mail address: Luis_Guijarro@Mat.UCM.Es

FB MATHEMATIK

Uni. Goettingen

Bunsenstrasse 3

37073 GoetTingen

Germany

E-mail address: schick@uni-math.gwdg.de

Department of Mathematics

UNIVERSITY OF OKLAHOMA

NORMAN OK 73019 
E-mail address: gerard@math.ou.edu 